\title{
ELECTRICAL SIGNS IN THE DIAGNOSIS OF CARPAL TUNNEL AND RELATED SYNDROMES*
}

\author{
BY \\ JOHN A. SIMPSON

\begin{abstract}
From the Neurological Research Unit and the Department of Applied Electrophysiology, National Hospital,
\end{abstract} \\ Queen Square, London
}

A common problem in clinical neurology is the differential diagnosis of the cause of tingling or numbness of fingers which may be associated with weakness of the small muscles of the hand. There is general recognition that the syndrome may result from compression of nerve roots or peripheral nerves supplying the hand, or of the blood vessels supplying the affected nerves, and that compression may occur at various sites in the spine (Brain, Northfield, and Wilkinson, 1952), thoracic outlet (Walshe, 1951), and more peripherally. The median nerve is commonly compressed in the carpal tunnel on the flexor aspect of the wrist (Kremer, Gilliatt, Golding, and Wilson, 1953) and the diagnosis is of considerable importance in view of the efficacy of decompression of the carpal tunnel (Brain, Wright, and Wilkinson, 1947).

Electromyographic diagnosis has hitherto depended on the demonstration of evidence of partial denervation confined to the muscles supplied by a particular motor nerve or one of its branches. This method has certain practical disadvantages. It may fail to demonstrate changes at an early stage which are diagnostic and it necessitates widespread exploration of the intrinsic muscles of the hand with a needle electrode.

Considering that the pathological basis of these syndromes is presumed to be a local ischaemic lesion of a peripheral nerve or one of its branches it seemed worth examining patients for phenomena known to be present in experimental ischaemia of short lengths of nerves. These are (1) diminished conduction velocity of the nerve impulse through the ischaemic segment, and (2) repetitive firing of motor nerve fibres after a single electric shock stimulus (Kugelberg and Cobb, 1951).

\section{Material and Methods}

Clinical colleagues were invited to refer for study patients in whom the diagnosis of carpal tunnel syndrome seemed probable. The survey was subsequently extended

* Based on a paper read before the Association of British Neurologists on November 11, 1955. to include patients in whom the diagnosis was less certain and confirmatory evidence was required. Seventeen patients were examined, two of whom had ulnar nerve lesions, one at the elbow and one of the deep branch at the wrist. Ten normal subjects, five patients with motor neuron disease, and 10 patients with various muscular diseases were examined as controls.

Cathodal stimulation was applied at various points along the course of the nerve with a brief condenser discharge (time constant $20 \mu \mathrm{sec}$.) which was triggered by the time-scale of the recording oscillograph at a point indicated on it. The stimuli were applied through an isolating transformer to reduce artefacts. An Ediswan E.E.G. electrode was used for the cathode and a large plate electrode over the upper arm as anode. Threshold or supramaximal stimuli (judged from the amplitude of the muscle action-potential) were applied once per second.

Electromyographic recordings were made through a coaxial needle electrode inserted into the abductor pollicis brevis or opponens pollicis for median nerve, and abductor digiti minimi or first dorsal interosseus muscle for ulnar nerve conduction studies. A surface electrode placed between stimulating cathode and needle electrode was connected to the earth terminal of the amplifier to diminish stimulus artefact in the records. The action potentials were amplified by a differential amplifier with an adequate frequency response, and displayed on one beam of a double-beam cathode ray oscillograph. The other beam was fed with a time-scale giving intervals of 1 and $10 \mathrm{~m} . \mathrm{sec}$. derived from a crystal oscillator.

Ten sweeps of the oscillograph beam were superimposed in the photographic record. The time-interval between the recorded stimulus artefact and the initial deflection of the summated muscle action-potential identified at maximum gain was measured at each site of stimulation. The length of nerve from stimulating cathode in each situation to the recording needle-electrode was estimated by surface measurement. Symmetrical points of stimulation and recording were compared in both arms. In a few instances the median and ulnar nerves of the same limb were compared.

\section{Results}

The latency of the earliest action potential recorded in the opponens pollicis of normal subjects 
when stimulating the median nerve just above the proximal crease on the flexor surface of the wrist was usually $3 \mathrm{~m} . \mathrm{sec}$. and never more than $5 \mathrm{~m} . \mathrm{sec}$. The latency from stimulation of the same nerve in the antecubital fossa to the same recording point was $8-12 \mathrm{~m} . \mathrm{sec}$, the variation depending mainly on the length of the limb. The time required for conduction of the impulse between the two points of stimulation can be obtained by subtraction. In 11 of the 15 patients in whom the carpal tunnel syndrome was thought to be present the latency of response was significantly increased (up to $14 \mathrm{~m} . \mathrm{sec}$. at the wrist) on one or both sides (Table I). Typical samples of the type of record from which the measurements were made are shown in Fig. 1.

Calculation shows that the delay can be entirely accounted for by slowed conduction distal to the wrist stimulation-site, which might be due to physiological abnormality in the carpal tunnel or delay in neuromuscular transmission. Owing to physical limitations it is impossible to decide between these alternatives by stimulation distal to the carpal tunnel. A comparable experiment, however, was possible in a case of right ulnar nerve paresis due to chronic trauma at the elbow, which had a permanent flexion deformity and increased carrying angle following faulty reunion of a fracture (Fig. 2).
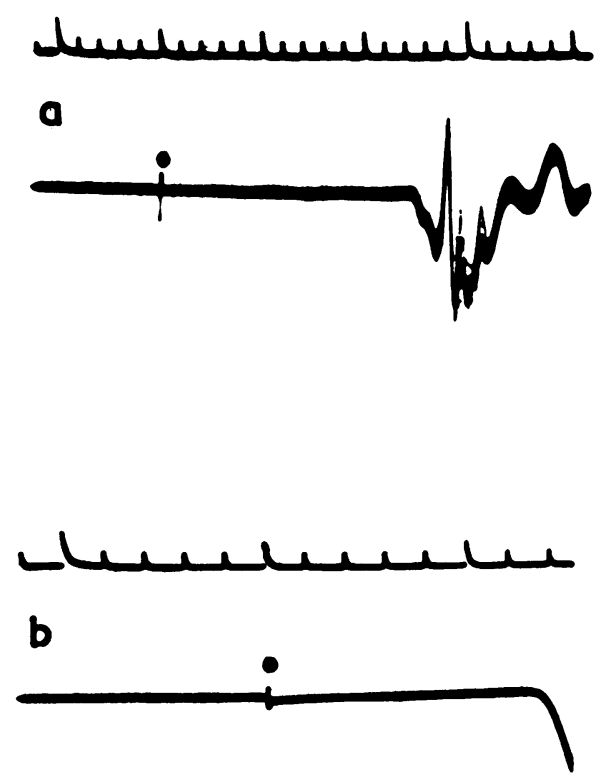

FIG. 1.-Case 13: Right carpal tunnel syndrome.

Ten superimposed action-potentials of right $(a, b)$ and left $(c, d)$ abductor pollicis brevis evoked by supramaximal stimulation of the appropriate median nerve at the elbow $(a$ and $c$ ) and at the wrist $(b$ and $d)$. Stimulus marked by spot. Time marks 1 and $5 \mathrm{~m} . \mathrm{sec}$. in each record (scale varies).
TABle I

DISTANCE FROM CATHODE TO RECORDING ELECTROH WITH LATENCY OF FIRST EVOKED ACTION POTENTIA
IN ABDUCTOR POLLICI BREVIS OR OPPONENS POLLIES IN CASES OF CARPAL TUNNEL SYNDROME

\begin{tabular}{|c|c|c|c|c|c|c|c|c|}
\hline \multirow{3}{*}{ Case } & \multicolumn{4}{|c|}{ Right Median } & \multicolumn{4}{|c|}{ Left Median } \\
\hline & \multicolumn{2}{|c|}{ Elbow } & \multicolumn{2}{|c|}{ Wrist } & \multicolumn{2}{|c|}{ Elbow } & \multicolumn{2}{|c|}{ Wrist $\lesssim$} \\
\hline & $\mathrm{cm}$. & m.sec. & $\mathrm{cm}$. & m.sec. & $\mathrm{cm}$. & m.sec. & $\mathrm{cm}$. & m.s. \\
\hline $\begin{array}{c}\text { Normal } \\
1 \\
2 \\
2 \\
3 \\
4 \\
5 \\
6^{*} \dagger \\
7 \\
8 \\
9 \\
10 \\
11^{*} \\
12 \\
13 \\
14 \\
15^{*}\end{array}$ & $\begin{array}{l}\overline{28.5} \\
28 \\
34 \\
28 \cdot 5 \\
31 \\
\overline{27} \\
33 \\
32 \\
28 \\
32 \cdot 5 \\
37 \\
28 \\
28 \cdot 5 \\
30\end{array}$ & $\begin{array}{c}8-12 \\
17 \\
12 \\
12 \cdot 5 \\
11 \\
11 \\
\frac{9}{9} \\
18 \cdot 5 \\
9 \\
9 \\
15 \\
13 \\
12 \cdot 5 \\
9 \\
20\end{array}$ & $\begin{array}{l}\overline{6} \\
5 \\
7 \\
5 \cdot 5 \\
5 \\
5 \\
7 \cdot 5 \\
6 \\
6 \\
7 \cdot 5 \\
8 \\
6 \cdot 5 \\
6 \\
6\end{array}$ & $\begin{array}{c}3-5 \\
11 \dagger \\
8 \\
6 \cdot 5 \\
6 \\
5 \cdot 5 \\
-5 \\
5 \cdot 5 \\
3 \cdot 5 \\
5 \\
10 \cdot 5 \\
7 \\
7 \\
4 \\
14\end{array}$ & $\begin{array}{l}28 \cdot 5 \\
28 \\
33 \\
29 \cdot 5 \\
30 \cdot 5 \\
25 \\
30 \\
34 \cdot 5 \\
33 \cdot 5 \\
32 \cdot 5 \\
34 \\
27 \\
29 \\
29\end{array}$ & $\begin{array}{c}10 \cdot 5 \\
10 \\
13 \\
8 \cdot 7 \\
9 \cdot 5 \\
13 \\
19 \\
15 \\
9 \\
\frac{1}{18 \cdot 5} \\
10 \\
7 \cdot 5 \\
9 \cdot 5 \\
18\end{array}$ & $\begin{array}{l}-\overline{6} \\
5 \\
6 \cdot 5 \\
6.5 \\
6 \cdot 5 \\
5 \\
5 \\
8 \\
5 \cdot 5 \\
5 \cdot 5 \\
6 \\
6 \cdot 5 \\
6 \\
6\end{array}$ & $\begin{array}{r}3 \\
4 \\
4 \\
7 \\
47 \\
58 \\
8 \\
14 \\
10 . \\
4 \cdot 8 \\
13 \\
4.8 \\
3.5 \\
43.8\end{array}$ \\
\hline
\end{tabular}

*Normal conduction velocity demonstrated in ulnar nerve. $\omega$ † Significant peripheral delay shown by figures in italics.

The ulnar nerve could be stimulated at two poinits in the upper arm proximal to the lesion and at two points in the forearm distal to it and the latengies of response of the earliest unit recorded abductor digiti minimi compared with thoge recorded in a similar way from the left arm (Fi 3 ).
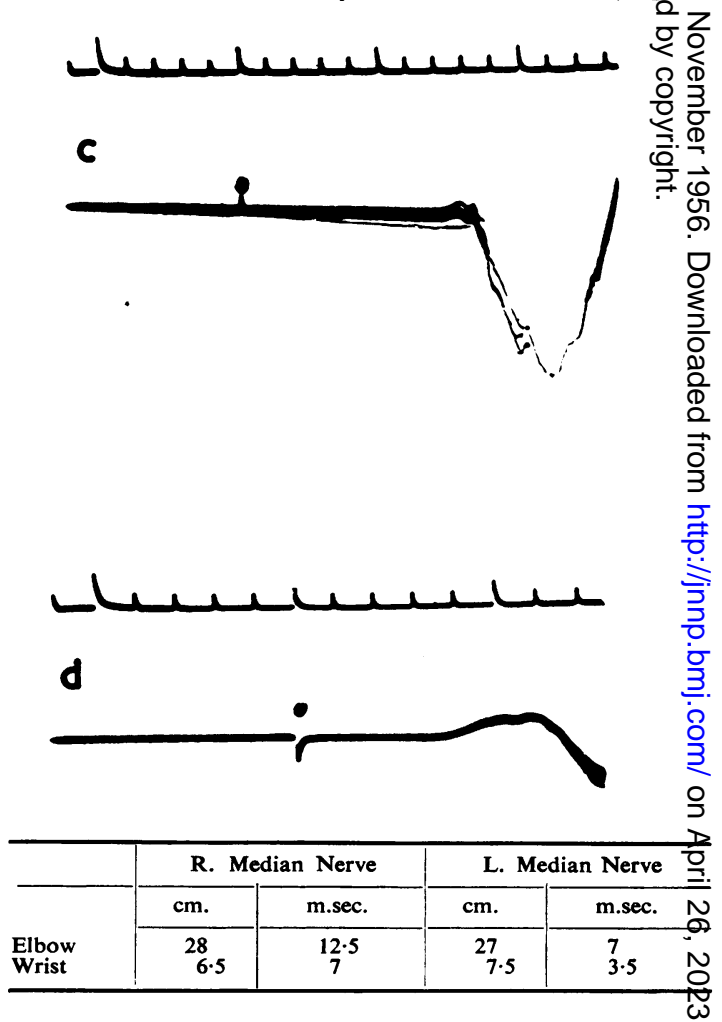
The latencies were virtually identical when each side was stimulated at the wrist and upper forearm, but were doubled on the affected side for stimulation proximal to the lesion. In this case the increased latency must be attributed to decreased conduction velocity in the affected segment of nerve and it can be presumed that the same explanation holds in the carpal tunnel syndrome.

Pressure lesions of the ulnar nerve occur less commonly in the hand and tend to be situated distal to the branch to the hypothenar muscles (Seddon, 1952). One case of deep branch neuritis of the right ulnar nerve with sparing of hypothenar muscles was examined. The ulnar nerve was stimulated at three points and action potentials recorded in the first dorsal interosseous and abductor digiti minimi (the latter path being $4 \mathrm{~cm}$. shorter by surface measurement). Control measurements on the right median nerve, to the opponens pollicis, resembled those of the ulnar branch to the abductor digiti minimi. The conduction velocity of fibres to the abductor digiti minimi was normal and compared closely with that of the median nerve of the same limb but there was a marked delay peripheral to the wrist in the fibres to the first dorsal interosseous muscle (Table II).

TABLE II

CONDUCTION TIME IN ULNAR DEEP-BRANCH NEURITIS

\begin{tabular}{|c|c|c|c|c|c|}
\hline \multicolumn{4}{|c|}{ Right Ulnar } & \multirow{2}{*}{\multicolumn{2}{|c|}{$\frac{\text { Right }}{\substack{\text { Opponens } \\
\text { Pollicis }}}$}} \\
\hline \multicolumn{2}{|c|}{$\begin{array}{l}\text { First Dorsal } \\
\text { Interosseous }\end{array}$} & \multicolumn{2}{|c|}{$\begin{array}{c}\text { Abductor Digiti } \\
\text { Minimi }\end{array}$} & & \\
\hline $\mathrm{cm}$. & m.sec. & $\mathrm{cm}$. & m.sec. & $\mathrm{cm}$. & m.sec. \\
\hline $\begin{array}{l}38 \\
21 \\
12\end{array}$ & $\begin{array}{l}19: 30^{*} \\
15: 26^{*} \\
12: 23^{*}\end{array}$ & $\begin{array}{r}34 \\
17 \\
8\end{array}$ & $\begin{array}{l}8 \cdot 5 \\
4 \\
3\end{array}$ & $\frac{32}{5 \cdot 5}$ & $\frac{8}{3 \cdot 5}$ \\
\hline
\end{tabular}

* The initial unit and a later recognizable unit. Each was delayed in comparison with the abductor digiti minimi and each unit maintained the same temporal dispersion.

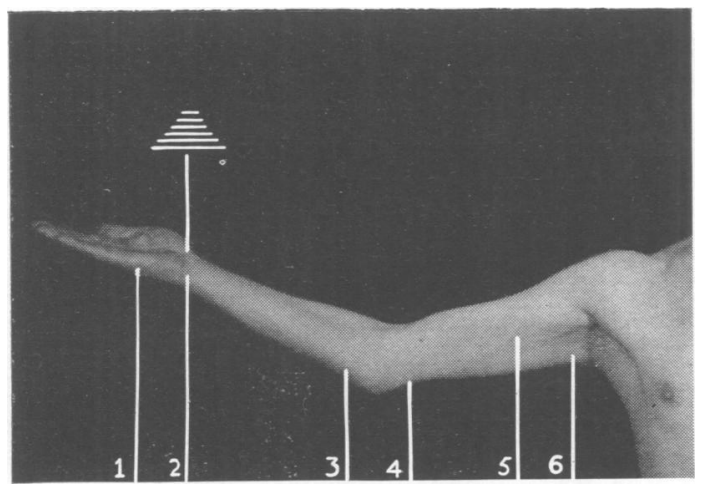

FIG. 2.-Patient with chronic traumatic ulnar neuritis at right elbow, illustrating method used. Needle electrode in abductor digiti minimi (1) and stimulating cathode applied in turn to points 2 , 3,4 , and 5 over the ulnar nerve. Stimulating anode over upper arm (6) and earthed electrode just proximal to needle electrode.
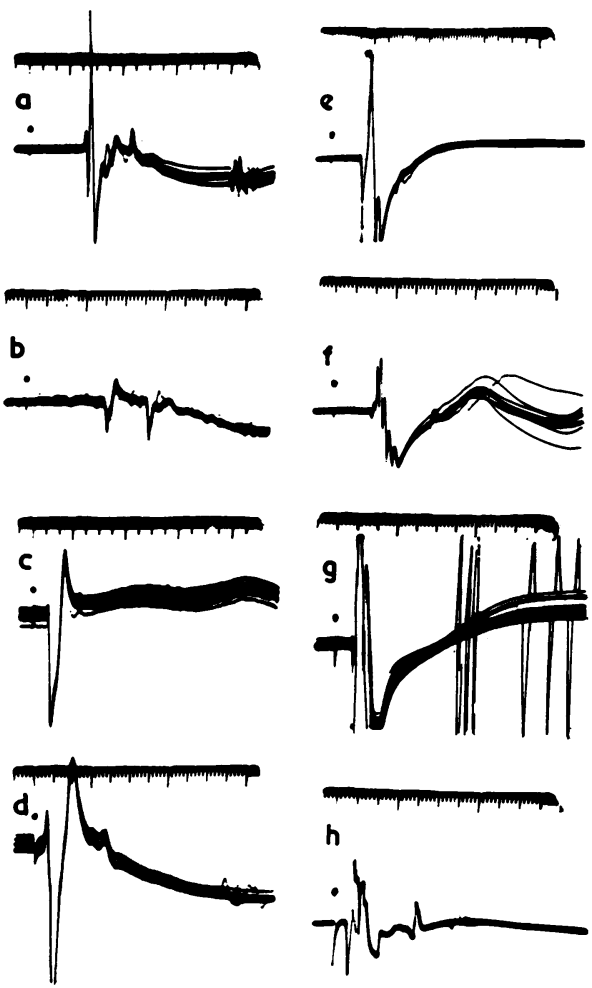

Fig. 3.-Case shown in Fig. $2 a-d$ right ulnar, $e-h$ left ulnar nerve. Recording from abductor digiti minimi of each hand. Time marks 1 and $5 \mathrm{~m} . \mathrm{sec}$. (scale varies).

\begin{tabular}{l|c|c|c|c}
\hline & \multicolumn{2}{|c|}{ R. Ulnar Nerve } & \multicolumn{2}{c}{ L. Ulnar Nerve } \\
\cline { 2 - 5 } & $\mathrm{cm}$. & $\mathrm{m.sec}{ }^{*}$ & $\mathrm{~cm}$. & $\mathrm{m.sec}{ }^{*}$ \\
\hline Axilla & $43 \cdot 5$ & $20 \cdot 5$ & $46 \cdot 5$ & 10 \\
Above elbow & 37 & $17 \cdot 5$ & 39 & $8 \cdot 7$ \\
Below elbow & 17 & 5 & 17.5 & $3 \cdot 5$ \\
Wrist & 6.5 & $2 \cdot 5$ & 6.5 & $2 \cdot 5$ \\
\hline
\end{tabular}

*Latencies measured on larger scale records than those reproduced.

Five patients with carpal tunnel syndrome were re-examined after operative decompression of the median nerve (Table III and Fig. 4). The shortest latency in the abductor pollicis brevis or opponens pollicis from supramaximal stimulation just proximal to the flexor retinaculum was compared with the pre-operative measurement. In Cases 7 and 8 the nerve causing most pain was decompressed though conduction velocity was less impaired than on the other side. In Case 3 the latter had been previously decompressed. Two cases showed no change in conduction time one month after operation and no further examination was obtained. In the other three cases the latency of response returned to normal though in one case the prolongation was increased during the first fortnight and recovery was not measurable until two months after operation. 
TABLE III

EFFECT OF OPERATIVE DECOMPRESSION IN CARPAL TUNNEL SYNDROME

\begin{tabular}{|c|c|c|c|}
\hline \multirow{2}{*}{ Case } & \multicolumn{2}{|c|}{ Operated Nerve } & \multirow{2}{*}{ “ Control " $\begin{array}{c}\text { Nerve } \\
(\mathrm{m} . \mathrm{sec} .)\end{array}$} \\
\hline & $\begin{array}{l}\text { Before } \\
\text { (m.sec.) }\end{array}$ & $\underset{\text { (m.sec.) }}{\text { After }}$ & \\
\hline 1 & 11 & $\begin{array}{l}6 \cdot 5^{*} \\
5 \cdot 5 \dagger\end{array}$ & 4 \\
\hline 2 & 8 & $\begin{array}{r}5 \cdot 0 \pm \\
10 \\
8 \cdot 5+ \\
6.5 t\end{array}$ & 4 \\
\hline $\begin{array}{l}3 \\
7 \\
8\end{array}$ & $\begin{array}{l}7 \\
5 \\
8.5\end{array}$ & $\begin{array}{l}4.5 \dagger \\
5.5 \dagger \\
8 \cdot 5 \dagger\end{array}$ & $\begin{array}{l}6 \cdot 5 \\
14 \\
10\end{array}$ \\
\hline
\end{tabular}

*2 weeks after operation. $\$ 8-12$ weeks after operation.
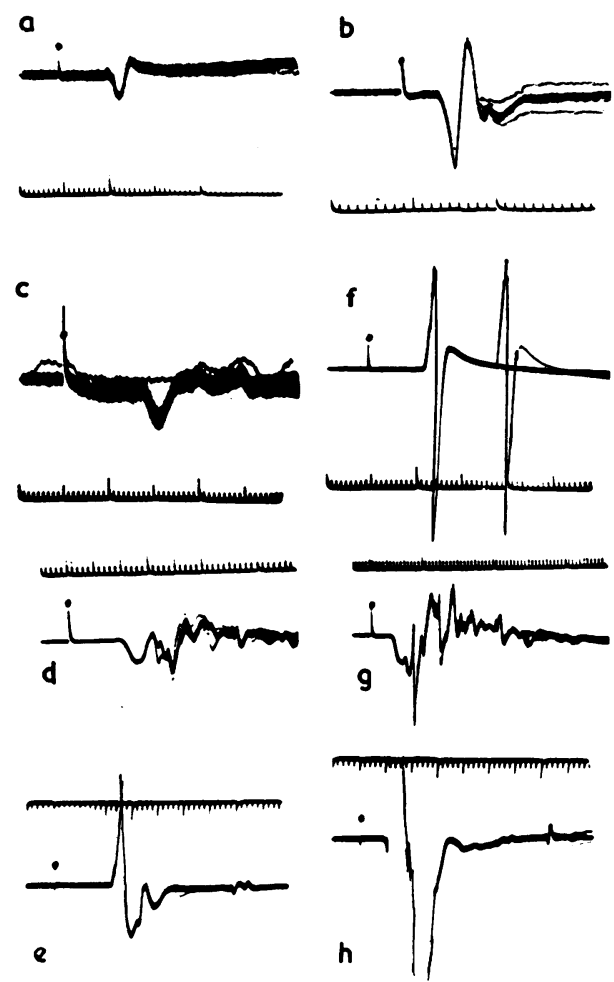

Fig. 4.-Case 1, right carpal tunnel syndrome. Effect of decompression.

$a-b$. Left median nerve to abductor pollicis brevis ( $a=$ elbow; $b=$ wrist)

$c-h$. Right median nerve to abductor pollicis brevis $(c-e=$ elbow; $f-h=$ wrist).

Compare $c, f$ (pre-operative) with $d, g$ ( 2 weeks) and $e, h$ (3 months). Note progressive reduction in latency and temporal dispersion of muscle action-potential, and repetitive firing $(f)$. (cf. Table II).

Time marks 1 and 5 or $10 \mathrm{~m} . \mathrm{sec}$. (scale varies).

Delay in Slower Fibres. - The configuration of the summated action-potential of muscle, whether recorded by surface electrodes or as in the present study, suggests that motor units are not quite synchronously innervated by a supramaximal shock applied to a point on the motor nerve. The sump. mated muscle action-potential broadens and $\mathrm{d} g-$ creases in amplitude as the stimulating electrode $\Phi_{8}$ moved proximally along the nerve (Bolzani, 1954 . The asynchrony increases in the carpal tunnes syndrome (e.g., Fig. 4) so that the muscle action potential may last for $50 \mathrm{~m} . \mathrm{sec}$. or longer (norm? about $20 \mathrm{~m} . \mathrm{sec}$.) and become highly polyphas The disintegration may allow individual motor units to be recognized later than the shortest latency un $\overrightarrow{\vec{\delta}}$, particularly when a needle electrode is used. After decompression of the nerve the scatter of latencies reduced and the summated muscle action potenti商 regains its normal form (Fig. 4). The possibility suggests itself that the increased latency to the earliest-firing unit in the ischaemic nerve is due to block of only the largest (and presumably fastest) fibres but this would not explain the great increase of the total duration of the complex. Furthermores in some cases of carpal tunnel syndrome, as experimental ischaemia of a segment of ner (Kugelberg and Cobb, 1951), the whole complect is delayed without much alteration in its configuration. Thus it seems likely that decreased conduction velocity affects all sizes of fibre.

Repetitive Firing.-In a considerable experiōnce of this type of investigation the author has never recorded repetitive firing of motor units in normil subjects, but this phenomenon was present in seve of the 15 patients with carpal tunnel syndrere (Figs. 4 and 5). The occurrence of this is restily recognized by observation of the oscilloscope screesp or by listening to the monitoring loudspeaker. P Fig. 4, where the whole complex within range of the needle fired twice during a single sweep (of the superimposed), or in Fig. 5, where a single (thresholfㄱ) unit is readily identifiable by its shape, the natufe of the "double" or " treble" unit recorded क्ष readily recognized, but repetitive firing occurring during the dispersed complex described above con be difficult to recognize, especially if the stimulus is not truly supramaximal. The superimpositiof of successive records is valuable since the repetitive firing occurs with only a fraction of the stimuli a its period shows considerable variability (Fig. 5).

Table I shows that bilateral conduction delay motor fibres was common, suggesting that the lesion may be bilateral though subjective symptoms afe only unilateral. Indeed the abnormality of conduction may be worse on the side with least sensopy disturbance and repetitive firing was sometimes found in the muscles supplied by the median nerzie on that side. An excellent example of repetitife firing (probably of the $F$ wave) (Magladery and McDougal, 1950) (Fig. $3 \mathrm{~g}$ ) was recorded from the 

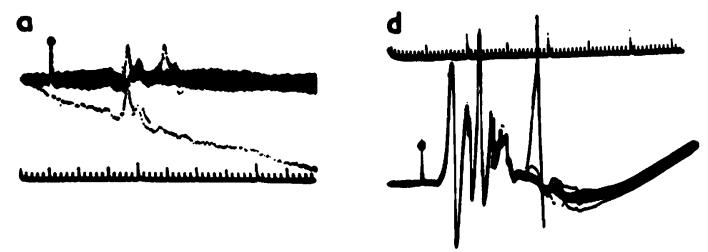

b

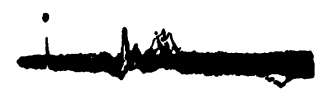

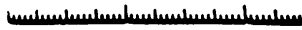

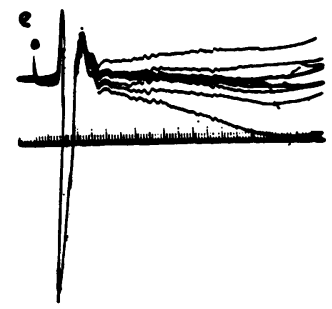

c

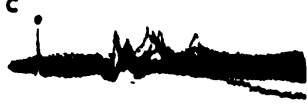

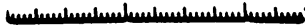

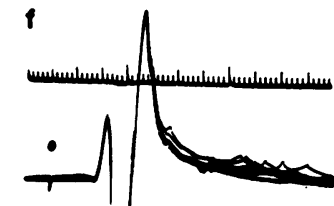

FIG. 5.- $a, b, c$, consecutive records of 10 superimposed responses from abductor pollicis brevis in carpal tunnel syndrome. A single unit, evoked by threshold stimuli, has a recognizable shape enabling its occasional repetitive firing (once or twice) to be identified. The period is variable $(b, c)$ and also in $d$ and $f$ (from other cases), but remarkably constant in $e$. Time marks 1 and 5 or $10 \mathrm{~m} . \mathrm{sec}$.

apparently unaffected side in the case of chronic traumatic ulnar neuritis. No explanation for this is apparent.

\section{Discussion}

The present investigation was suggested by a personal re-investigation of the effects of local compression of a peripheral nerve as previously described by Kugelberg (1946) and Kugelberg and Cobb (1951). These authors have shown that during the application of compression the conduction velocity in motor nerve fibres progressively decreases in the compressed segment only and that during compression and for a short time afterwards repetitive firing of motor nerve fibres occurs either spontaneously or as the result of electrical stimulation. Kugelberg (1946) suggested that a similar phenomenon in sensory fibres would explain paraesthesiae and pain occurring in certain circumstances.

It seemed probable that the same changes would be found in pathological processes associated with localized ischaemic changes in peripheral nerve. The expected modification of conduction and the presence of repetitive firing were observed. These changes have not hitherto been recorded in natural pathological states. The observation that conduction is normal distal to the lesion and the recovery after operation make it unlikely that the slow conduction occurs in regenerating fibres. The results of this investigation have not been tabulated as conduction velocities calculated from the distances and latencies because the possible error involved in the calculation is considerable. The length-measurement made on the surface is very approximate and the surface area of the stimulating cathode (diameter $1 \mathrm{~cm}$.) is relatively large and its zone of effective influence in the tissues unknown but probably larger, particularly with the supramaximal stimulation considered essential. Thus the " effective cathode" at the nerve may be more than a centimetre in either direction from the centre of the electrode. Time measurement is probably not accurate to more than $0.5 \mathrm{~m} . \mathrm{sec}$., as it is often difficult to define exactly where the recorded action potential begins to deviate from the base line. A rough calculation shows that with a "measured length" of nerve of $20 \mathrm{~cm}$. and " measured time" of $5 \mathrm{~m} . \mathrm{sec}$. the calculated velocity of conduction could be 35-47 m.p.s. Even then the calculated figure represents an average based on the assumption of constant velocity of conduction in the measured length of nerve whereas in fact there is evidence that normal peripheral nerve conducts with decreasing velocity as it extends peripherally and this difference is exaggerated by cooling of the periphery (Baxt and Helmholtz, 1870). It is submitted that the method of comparison of corresponding nerves of each upper limb, stimulated in the same way at symmetrical points and recorded under the same conditions of temperature, provides satisfactory criteria of difference from the normal without introducing quantitative concepts with high inherent fallacies.

The present investigation was not designed to test the reliability of these changes as a diagnostic examination. To assess the significance of normal findings and the correlation of the electrical signs with the symptoms in the early case of carpal tunnel syndrome, a long-term follow-up of cases in which no conduction abnormality was detected and sequential examination of early untreated cases would be necessary. Nevertheless, the incidence of positive findings in the small number of patients investigated suggests that the changes described in this paper will be of value as a confirmatory test.

\section{Summary}

Slowed conduction has been demonstrated in the presumed ischaemic section of the median nerve in 11 of 15 cases of carpal tunnel syndrome (bilateral 
in four cases) and in three cases this was shown to return towards normal after operative decompression.

Repetitive firing of motor units after single shock stimulation was present in seven of 15 cases. Increased temporal dispersion of the summated actionpotential of a muscle supplied by the compressed nerve returned to normal after decompression.

In one patient with chronic traumatic neuritis of the ulnar nerve at the elbow the slowed conduction was shown to be confined to the damaged segment of nerve.* In a patient with neuritis of the deep branch of the ulnar nerve it was demonstrated that the branch to abductor digiti minimi escaped the conduction block which was found in fibres to the first dorsal interosseous muscle.

* Since this paper was written the findings have been confirmed in three further cases.
I should like to thank Dr. W. A. Cobb and Mr. 局. Morton for providing facilities and technical assistane्, and Drs. E. A. Carmichael, R. Gilliatt, and M. Kremor for permitting me to examine their patients. Discussiofs with Dr. G. Dawson were most helpful. The work wass done during the tenure of an M.R.C. fellowship om clinical research.

\section{REFERENCES}

Baxt, N., and Helmholtz, H. (1870). Mber. preuss. Akad. Wi Berlin, p. 184

Bolzani, L. (1954). Schweiz. Arch. Neurol. Psychiat., 74, 148.

Brain, W. R., Northfield, D., and Wilkinson, M. (1952). Bră. $75,187$.

Kugelberght, A. D., and Wilkinson,

Kugelberg, E. (1946). Brain, 69, 310. - , and Cobb, W. A. (1951). Journal of Neurology, Neurosurg $\frac{\omega}{2 y}$ and Psychiatry, 14, 88.

Kremer, M., Gilliatt, R. W., Golding, J. S. R., and Wilson, T. Z્. Magladery, J. W., and McDougal, D. B. (1950). Bull. Johns Hopk. Hosp. 86, 265.

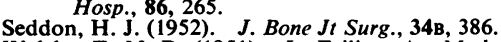

Walshe, F. M. R. (1951). In Feiling, A. Modern Trends in Neurotogy. Butterworth, London. 